\title{
Comparative Study of Volatility Forecasting Models: The Case of Malaysia, Indonesia, Hong Kong and Japan Stock Markets
}

\author{
San K. Lee, Lan T. P. Nguyen \\ Multimedia University, Cyberjaya, Malaysia \\ Malick O. Sy \\ Royal Melbourne Institute of Technology, Melbourne, Australia
}

\begin{abstract}
This paper aims to investigate the effectiveness of four volatility forecasting models, i.e. Exponential Weighted Moving Average (EWMA), Autoregressive Integrated Moving Average (ARIMA) and Generalized Auto-Regressive Conditional Heteroscedastic (GARCH), in four stock markets Indonesia, Malaysia, Japan and Hong Kong. Using monthly closing stock index prices collected from 1st January 1998 to 31st December 2015 for the four selected countries, results obtained confirm that volatility in developed markets is not necessarily always lower than the volatility in emerging markets. Among all the three models, GARCH $(1,1)$ model is found to be the best forecasting model for stock markets in Malaysia, Indonesia, and Japan, while EWMA model is found to be the best forecasting model for Hong Kong stock market. The outperformance of GARCH $(1,1)$ found supports again what is found in Minkah (2007).
\end{abstract}

Keywords: volatility forecasting models, $\operatorname{GARCH}(1,1)$, EWMA, ARIMA, effectiveness, emerging countries

\section{Introduction}

Study on the implied volatility of stock markets has been growing over the past decades. However, to forecast well stock market volatility, having a reliable forecasting model is essential. Past studies fail to arrive at a common conclusion in terms of forecasting models for a specific stock market (Adebayo \& Sivasamy, 2014; Lim \& Sek, 2013; Ladokhin, 2009; Febrian, 2006; Schwert, 1990), which may be due to the variation in their selected data samples and study periods (Wilhelmsson, 2006). It is a common belief that stock markets in developed countries are less volatile as compared to those in developing countries due to the weaker economic fundamentals often present in emerging markets. Given the complexity of the study on volatility, this study attempts to exaimine whether volatility in emerging markets is indeed always higher as compared to that in developed markets, especially in Asia region. Four Asian countries are thus selected for this study, among which two (Indonesia and Malaysia) are representatives for emerging economies and two (Japan and Hong Kong) are representatives for developed economies. To test the effectiveness of three common volatility forecasting models, i.e., Exponential Weighted Moving Average (EWMA), Autoregressive Integrated Moving

San K. Lee, Faculty of Management, Multimedia University, Cyberjaya, Malaysia.

Lan Thi Phuong Nguyen, Ph.D., senior lecturer, Faculty of Management, Multimedia University, Cyberjaya, Malaysia.

Malick Ousmane Sy, Ph.D., professor of Finance, School of Economics, Finance and Marketing, RMIT, Melbourne, Australia.

Correspondence concerning this article should be addressed to Lan Thi Phuong Nguyen, Faculty of Management, Multimedia University, Jalan Multimedia, Cyberjaya, 63100 Malaysia. 
Average (ARIMA), and Generalized Auto-Regressive Conditional Heteroscedastic (GARCH), monthly stock prices are collected from 1st January 1998 to 31st December 2015 for the four-mentioned Asian countries.

The remainder of the paper is structured as follows: Section two presents the literature review related to the volatility forecasting. Section three elaborates on the methodology and econometric procedures adopted in this study. Section 4 discusses the empirical findings. In the final section-Section five-key conclusions are given.

\section{Literature Review}

Past studies have discussed a number of volatility forecasting models such as Naïve forecasting model, Autoregressive Moving Average (ARMA) model, smoothing method, Holt-Winters, Exponential Weighted Moving Average (EWMA), and GARCH models. In Schwert's (1990) study, the moving average and smoothing model is found to be superior to other methods, while GARCH $(1,1)$ model is found to be the poorest model. In terms of the Exponential Weighted Moving Average (EWMA) model, Tse (1991) found that this model is the best volatility forecasting model for both Japanese and Singapore stock markets, followed by the stochastic volatility model. In terms of the forecasting abilities, Febrian (2006) found that the combination of an autoregressive model and a volatility model such as GARCH would result in a better model which could allow volatility forecasting for the Jakarta Stock Exchange (JSX) to be more accurate. The author examined four different models, i.e., exponential smoothing, ARIMA model, EWMA model, ARCH model, and GARCH model, in his study. However, Minkah $(2007)$ found that GARCH $(1,1)$ appears to be more superior as compared to the historical standard deviation, exponentially weighted moving average model. However, its forecasting accuracy demonstrated some reservations as it is dominated by the non-linear least squares model. Ladokhin (2009) examined the effectiveness of nine volatility forecasting models for the New Zealand stock market. The author found the exponential smoothing model appears to be the best volatility forecasting model as indicated by the mean absolute error (MAE) statistics. When measuring the impact of the 2008 global financial crisis on the Malaysian stock market, Angabini and Wasiuzzaman (2010) find that AR(4) and $\operatorname{GARCH}(1,1)$ are best forecasting models for their study period of 2000-2010. In Kosapattarapim, Lin, and McCrae (2011), volatility estimates were used as the best-fitted model for Thailand, Malaysia, and Singapore stock exchanges. According to their results, a model which is based on AIC criterion is not necessarily a best-fitted model in terms of error measures such as Mean Squared Error (MSE) and Mean Absolute Error (MAE). However, they concluded that a best-fitted model is still able to give a sensible predictability on volatility. The authors also employed GARCH (p, q) model to forecast volatility with six types of error distributions, i.e., normal distribution, skewed normal distribution, student t-distribution, skewed student-t-distribution, generalized error distribution (GED), and Skewed Generalized Error Distribution. Their results showed that $\operatorname{GARCH}(1,3), \operatorname{GARCH}(1,1)$, and $\operatorname{GARCH}(2,1)$ are the best forecasting models for Thailand, Malaysia, and Singapore, respectively. In another study by Lim and Sek (2013), volatility of the Malaysia stock market was examined with the use of three models: GARCH, TGARCH, and EGARCH for a period of 1990-2010. To observe the impact of the Asian financial crisis, the authors constructed three sub-sample periods: the pre-crisis period of 2 January 1990 to 30 June 1997, the crisis period of 1 July 1997 to 30 September 1998, and the post-crisis period of 1 October 1998 to 30 December 2010. Using error measures such as Mean Squared Error (MSE), Root Mean Squared Error (RMSE), and Mean Absolute Percentage Error (MAPE), results obtained show that performance of each model varies during the study period. However, when 
using other error measures such as GARCH and TGARCH, stock market volatility seems to perform better, from which GARCH $(1,1)$ provides the most significant results. One of the studies using ARIMA model was done by Adebayo and Sivasamy (2014). ARIMA model is commonly used as a volatility forecasting model for stock markets. Using different selection criteria, i.e., AIC, BIC, HQC, RMSE, and MAE, the authors find that ARIMA $(3,1,1)$ and ARIMA $(1,1,4)$ models are best forecasting models for Botswana and Nigeria stock markets, respectively. Using the same Parkinson, Garman and Klass, Rogers and Satchell estimators, Yarovaya, Brzeszczyński and Lau (2016) attempt to examine if results on volatility spill overs across six different Asian stock markets when using different volatility estimators. The authors found that Hong Kong, South Korea, and Taiwan are net-contributors while Japan, Singapore, and China are the net-recipients. They concluded that empirical tests are susceptible to the choice of volatility estimators, therefore studies on volatility are suggested to include different volatility estimators to avoid possible biases in results.

With mixed conclusions given in the past studies, it is useful to examine further the effectiveness of three commonly used forecasting models such as ARIMA, EWMA, and GARCH for various stock markets in Asia.

\section{Methodology}

\section{Data Sample}

In this study, four Asian countries are chosen, among which two are emerging countries (Indonesia and Malaysia) and two are developed countries (Japan and Hong Kong), to be in our sample. Monthly closing prices for all stock indices (see Table 1) from the four countries are collected from the Bloomberg database for a period of 1998-2015, which consists of an in-the-sample period of 1998-2009 and an out-of-the-sample period of 2010-2015.

Table 1

Markets and Indices

\begin{tabular}{lll}
\hline Market & & Index \\
\hline Malaysia & MAL & FTSE KLCI index \\
Indonesia & INO & JKSE index \\
Hong Kong & HKG & Hang Seng index \\
Japan & JPN & Nikkei 225 index \\
\hline
\end{tabular}

Based on the closing prices, compounded monthly index returns are then computed as follows:

$$
r_{t}=\ln \frac{\text { Index }_{t}}{\text { Index }}
$$

where: $r_{t}=$ compounded monthly return; $I n=$ natural logarithmic function; index $x_{t}=$ current month index at time $t$; and index $x_{t-1}=$ previous month index at time $(t-1)$.

\section{Unit-Root Test}

Monthly return series for stock indices of the four selected countries are tested for a unit root with the use of Augmented Dickey-Fuller (ADF) test. The null and alternative hypotheses are stated for each time series data as follows:

$\mathrm{H}_{0}$ : monthly return series has a unit root

$\mathrm{H}_{1}$ : monthly return series has no unit root

Results obtained from the ADF test are presented in Table 2 as below. 
Table 2

Results Obtained From Augmented Dickey-Fuller Test at Level

\begin{tabular}{lll}
\hline Indices & $t$-statistic & Probability \\
\hline KLCI & -12.535 & 0.000 \\
JKSE & -11.686 & 0.000 \\
Hang Seng & -12.985 & 0.000 \\
Nikkei & -12.729 & 0.000 \\
\hline
\end{tabular}

As shown in Table 2, $p$-values of the $t$-statistics allow us to reject the null-hypothesis of "there is a unit root" for the series of monthly returns of the four stock indices, i.e., KLCI, JKSE, Hang Seng, and Nikkei at the $1 \%$ level of significance.

\section{Models}

Following past studies (Adebayo \& Sivasamy, 2014; Singh, 2015; Ladokhin, 2009; Tse, 1991; Schwert, 1990; Febrian, 2006), forecasting models that will be used in this study are Autoregressive Integrated Moving Average (ARIMA), Exponential Weighted Moving Average (EWMA), and $\operatorname{GARCH}(p, q)$.

\section{Autoregressive Integrated Moving Average (ARIMA)}

ARIMA model is a forecasting model that focuses on the analysis of the deviation in values rather than the actual data for each time series. In addition, the model also takes into account other characteristics of a time series such as its trend, seasonal effect, error, and non-stationary of the data. This model uses historical data to generalize a forecast, thus, increases the accuracy of its forecast, while keeping a minimum number of parameters which helps avoid the problem with multivariate models.

ARIMA model is expressed as:

$$
\operatorname{ARIMA}(p, d, q)
$$

where: $\mathrm{p}$ is the order of the autoregressive, $\mathrm{AR}(p) ; \mathrm{d}$ is the order of the differencing needed for stationary $(d) ; q$ is the order of the moving average process, MA $(q)$.

If there is no difference needed for the series, this model is also referred to as ARMA $(p, q)$ model. There are three main stages for an ARIMA modeling, i.e., the identification stage, estimation and diagnostic checking stage, and the forecasting stage. In the stage 1 -identification stage - a plotting of a time series is required to determine whether the series possesses a trend. If it does, a difference is needed to remove the trend. This process continuing till the series is stationary on its variance in order to produce a reliable ARIMA forecasting. Alternatively, taking a logarithm for a time series data can also transform it to a stationary time series data. In stage 2-estimation and diagnostic checking stage - a correlogram is generated to allow the $p$ order for the autocorrelation factor (ACF) and the $q$ order for the autocorrelation factor (PACF) in ARIMA model to be determined. If all bars are within the limit, the maximum lag can be tested is one. Alternatively, a unit root test can be carried out to find out if the time series data is stationary at the Level. ARIMA model will be then identified and tested and validated with normality, heteroscedasticity, and autocorrelation tests. In stage 3, with the specified ARIMA model, a forecasting model will be formed and tested. Details of the construction of this model with $\operatorname{AR}(p)$, differencing $(d)$, and MA $(q)$ as follows:

A $p$ th order autoregressive model, AR (p), in which has the general form of:

$$
y_{t}=\delta+\beta_{1} y_{t-1}+\beta_{2} y_{t-2}+\beta_{3} y_{t-3}+\cdots+\beta_{p} y_{t-p}+\varepsilon_{t}
$$

$Y_{t}$ is a dependant variable at time $t$. 
$Y_{t-1}, \ldots, Y_{t-p}$ are variables at respective time lag of the autoregressive.

$\varepsilon_{t}$ is an error term at time $t$.

A different order equation in which $y$ is denoted as the $d$ th difference of $Y$, where:

If $d=0: y_{t}=Y_{t}$

If $d=1: y_{t}=Y t-Y_{t-1}$

If $d=2: y_{t}=\left(Y_{t}-Y_{t-1}\right)-\left(Y_{t-1}-Y_{t-2}\right)=Y_{t}-2 Y_{t-1}+Y_{t-2}$

And a qth order moving average model, MA $(q)$, in which has the general form of

$$
Y_{t}=\mu+\varepsilon_{t}-\theta_{1} \varepsilon_{t-1}-\theta_{2} \varepsilon_{t-2}-\cdots-\theta_{q} \varepsilon_{t-q}
$$

where

$Y_{t}$ is dependant variable at time $t$.

$\mu$ is the constant mean of the process.

$Y_{t-1}, \ldots, Y_{t-p}$ represent variables at respective time lag of the autoregressive.

$\varepsilon_{t}$ is an error term at time $t$.

$\theta_{1}, \theta_{2}, \ldots, \theta_{q}$ are estimated coefficients.

$\varepsilon_{t-1}, \varepsilon_{t-2}, \ldots \varepsilon_{t-q}$ are errors at the previous time periods.

The general form of autoregressive moving average model $\operatorname{ARMA}(p, q)$ is:

$$
Y_{t}=\delta+\beta_{1} y_{t-1}+\beta_{2} y_{t-2}+\beta_{3} y_{t-3}+\cdots+\beta_{p} y_{t-p}+\varepsilon_{t}-\theta_{1} \varepsilon_{t-1}-\theta_{2} \varepsilon_{t-2}-\cdots-\theta_{q} \varepsilon_{t-q}
$$

The accuracy of the forecast value of $\operatorname{ARMA}(p, q)$ model can be tested either through generating of the static and dynamic forecast, error measures or via the setting of the prediction interval to determine whether the actual value falls within the interval values.

\section{Exponential Weighted Moving Average (EWMA)}

EWMA model averages time series data in a way that diminishes the data weight exponentially over time. This will allow possible impacts resulted from different events to be observed over time. Thus, EWMA model is considered to be a better model for volatility estimation. With EWMA model, higher weight is given to recent returns as opposed to older returns. The conduct of EWMA model consists of three steps. In the first step, monthly returns are computed by using Equation 1 as mentioned above. In the second stage, returns are squared by using the following formula:

$$
\sigma_{n}^{2}=\frac{1}{m} \sum_{i=1}^{m} u_{n-i}^{2}
$$

where: $u$ indicates the returns, and $m$ the number of days.

In third step, weight allocation will be done for all returns in an ascending order according to the time line. Based on the JP Morgan's Risk Metrics models, weights assigned to daily and monthly returns when estimating their volatilities are declining at a constant rate $\lambda$ of 0.94 and 0.97 , respectively. In this study, weight for the first return is computed as:

$$
1-0.97=0.03
$$

and weight for the second return is computed by taking the weight assigned to the first return multiplied by the constant rate of 0.97 as follows:

$$
0.03 \times 0.97=0.291
$$

Similar process will be done for the rest for other monthly returns in the sample. The total sum of all weights will be one as a result of this process. In the fourth step, all monthly returns are squared, multiplied by 
their respected weights, and summed up together. In the final step, using the following recursive formula of EWMA model to forecast volatility:

$$
\sigma_{n}^{2}=\lambda \sigma_{n-1}^{2}+(1-\lambda) r_{n-1}^{2}
$$

where: $r$ is the returns, lamda $(\lambda)$ here is a smoothing parameter in which weights are assigned to and it must be less than one.

\section{Generalized Auto-Regressive Conditional Heteroscedastic (GARCH)}

GARCH models are mostly preferred model to researchers when measuring volatility of stock market returns. Among all the GARCH models, $\operatorname{GARCH}(1,1)$ model is very popular, and it is expressed as follows:

$$
\sigma_{t}^{2}=\omega+\alpha \varepsilon_{t-2}^{2}+\beta \sigma_{t-1}^{2}
$$

where: $\sigma_{t}^{2}$ is conditional variance and $\omega, \alpha$ and $\beta \geq 0$. It is generally weakly stationary when $\alpha+\beta<1$. $\omega$ is the mean and $\varepsilon_{t-1}^{2}$ represents volatility from the previous period while $\sigma_{t-1}^{2}$ represent conditional variance from previous period.

\section{Testing for the Accuracy of a Forecasting}

To test for the accuracy for each of the forecasting models as mentioned above, three different error measures, i.e., mean absolute error (MAE), mean square error (MSE), and root mean squared error (RMSE) are used.

MAE is a statistical measure used to measure how close those obtained forecasted volatilities are to the actual volatilities. The expected error from the average forecasted volatility is computed as follows:

$$
M A E=\frac{1}{N} \sum_{i=1}^{N}\left|f_{i}-x_{i}\right|
$$

Mean Square Error (MSE) is used to measure how close the fitted line is to the data points, and is computed as follows:

$$
M S E=\frac{1}{N} \sum_{i=1}^{N}\left(\left|f_{i}\right|-x_{i}\right)^{2}
$$

And the Root Mean Square Error (RMSE) is computed as follows:

$$
R M S E=\sqrt{\frac{1}{N} \sum_{i=1}^{N}\left(\left|f_{i}\right|-x_{i}\right)^{2}}
$$

\section{Empirical Results}

\section{Descriptive Statistics of Emerging and Developed Market Return}

The prices of the four stock markets in our sample are plotted in Figure 1 below. It is clearly observed that stock index prices of the two developed markets (Hong Kong and Japan) exhibit higher patterns as compared to the other emerging markets (Indonesia and Malaysia) over the sample period of 1998-2015. In addition, a similar upward trend is observed for the two emerging stock indices, while a more fluctuating trend is found for both two other developed stock indices.

Table 3 presents descriptive statistics for all the four stock indices. Throughout the sample period, all stock indices of Japan, Hong Kong, Malaysia, and Indonesia exhibit all positive values during the sample period, from which higher mean values, i.e., 0.01 and 0.005 , are found for Indonesia and Malaysia, respectively.

As shown in Table 3, statistics for skewness, kurtosis, and Jacque-Berra all confirm that price distributions for all the four stock indices are not normally distributed. Values of the standard deviations obtained for 
Indonesia and Hong Kong stock markets are the highest, i.e., 0.078 and 0.069 , respectively, implying that these two are the most volatile markets among the four.

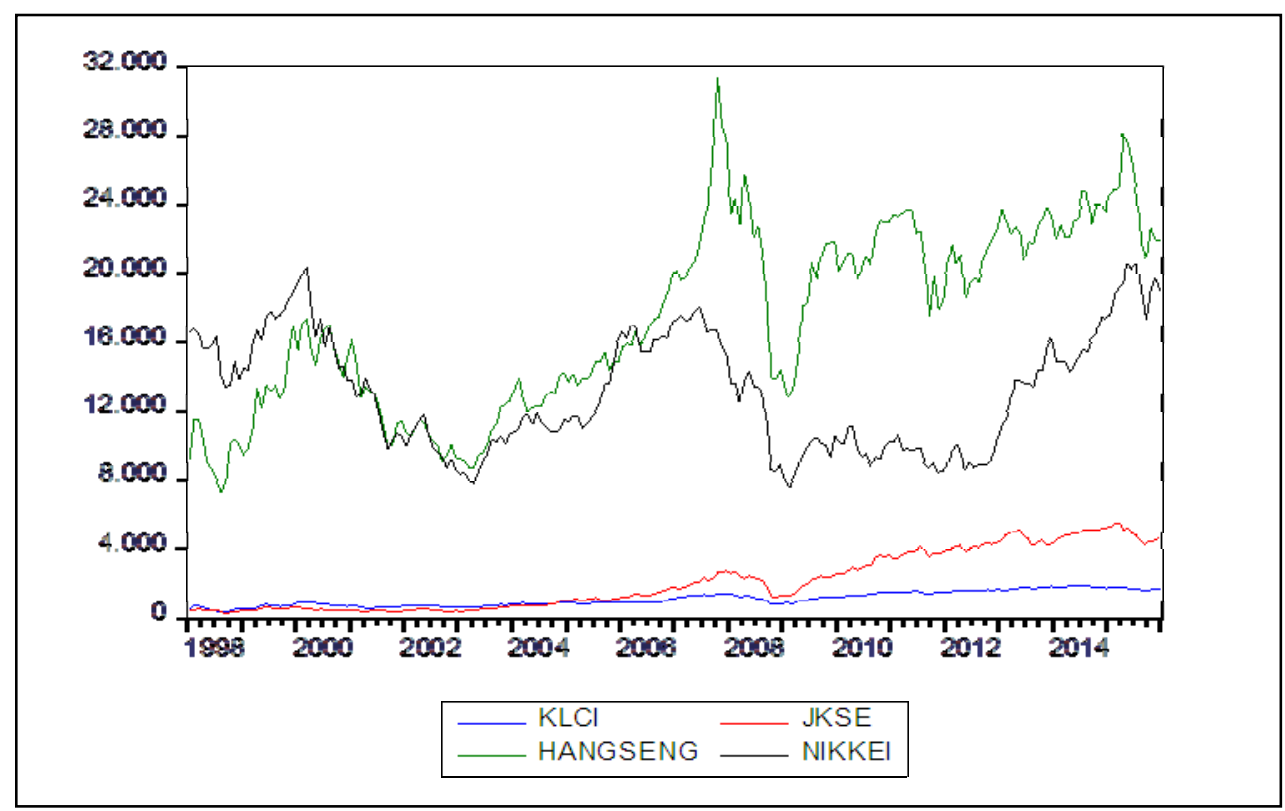

Figure 1. Prices for KLCI, JKSE, HANG SENG, and NIKKEI indices.

Table 3

Descriptive Statistics of Stock Market Indices Malaysia, Indonesia, Hong Kong, and Japan

\begin{tabular}{lllll}
\hline & Malaysia & Indonesia & Hong Kong & Japan \\
\cline { 2 - 5 } & FTSE KLCI index & JKSE index & Hang Seng index & Nikkei 225 index \\
\hline Mean & 0.005 & 0.010 & 0.004 & 0.001 \\
Median & 0.010 & 0.018 & 0.009 & 0.007 \\
Maximum & 0.290 & 0.250 & 0.253 & 0.121 \\
Minimum & -0.280 & -0.377 & -0.255 & -0.272 \\
Standard Deviation & 0.064 & 0.078 & 0.070 & 0.058 \\
Skewness & 0.302 & -0.928 & -0.026 & -0.743 \\
Kurtosis & 7.877 & 7.252 & 4.3257 & 4.516 \\
Jarque-Bera & 216.349 & 192.858 & 15.769 & 40.370 \\
Probability & 0.000 & 0.000 & 0.00 & 0.000 \\
Sum & 1.050 & 2.246 & 0.862 & 0.135 \\
Sum Sq. Dev. & 0.883 & 1.297 & 1.040 & 0.715 \\
Observations & 215 & 215 & 215 & 215 \\
\hline
\end{tabular}

As shown in Figure 2, the overall return trends for returns of the four indices are very similar during the sample period from 1998 to 2015. This may suggest that all four stock indices in our sample are influenced by all market factors in a very similar way.

\section{Results Obtained for ARIMA Model}

As all stock index (FTSE KLCI, JKSE, Hang Seng, and Nikkei) prices are all log-transformed to continuously compounded returns, the new return series do not exhibit any trend as shown in Figure 3. This confirms the results obtained from the ADF test conducted for a unit root as shown in Table 2 that all returns series of the four stock indices in the sample are stationary at level. 


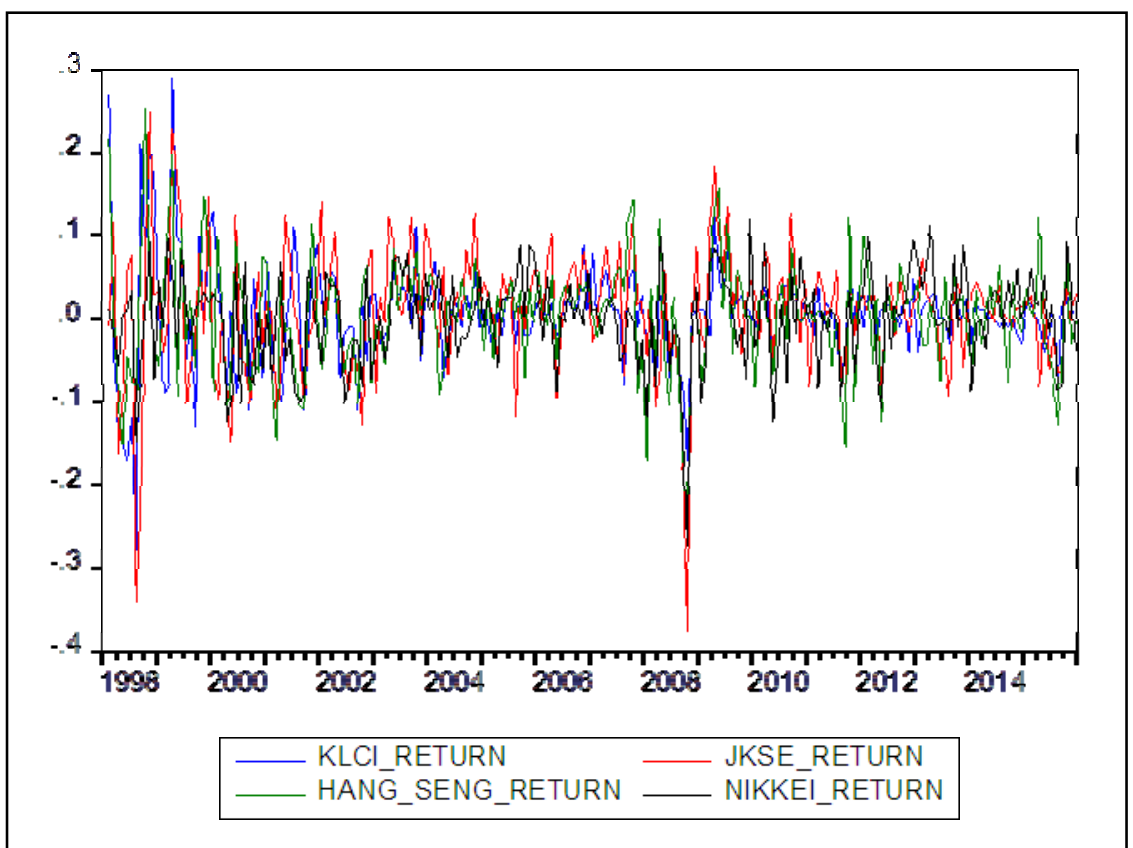

Figure 2. Overall stock market returns trend.

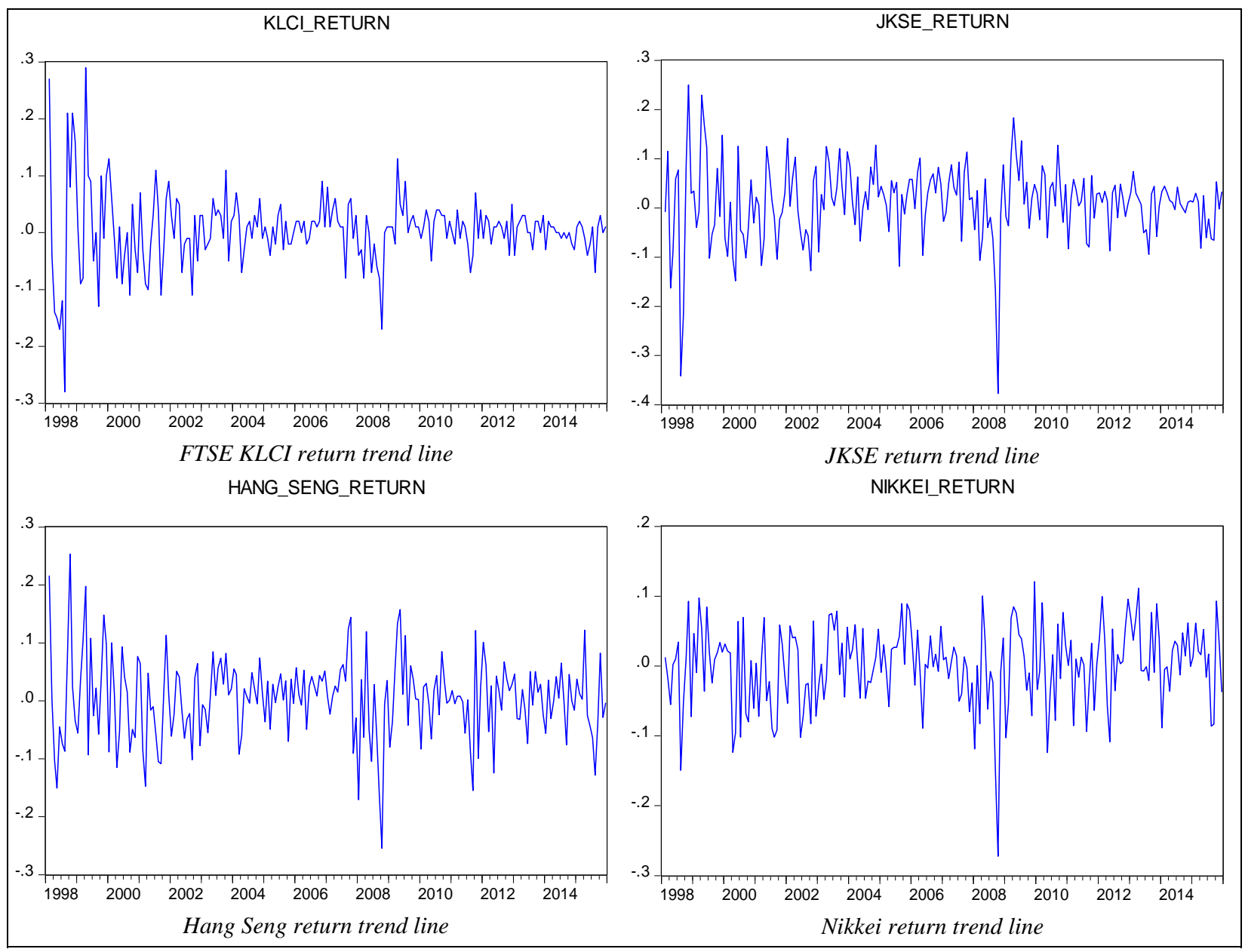

Figure 3. Return trend lines for the four markets in the sample. 
Based on the Akaike's information criterion (AIC), best ARMA models are then obtained for the four individual stock return indices as shown in Table 4 below.

Table 4

ARMA Model and AIC

\begin{tabular}{lll}
\hline Markets & ARMA model & AIC value \\
\hline FTSE KLCI & $(3,4)$ & -2.741 \\
JKSE & $(3,3)$ & -2.308 \\
Hang Seng & $(1,2)$ & -2.487 \\
Nikkei & $(1,0)$ & -2.845 \\
\hline
\end{tabular}

As shown in Table 4, best ARMA models found for the two emerging markets (Malaysia and Indonesia) all contain a lag of three, while best ARMA models found for the two developed markets (Hong Kong and Japan) all contain a lag of one. Thus, the ARMA forecasting models for the four stock indices are specified as follows:

For FTSE KLCI index:

$$
Y_{t}=\delta+\beta_{1} y_{t-1}+\beta_{2} y_{t-2}+\beta_{3} y_{t-3}+\varepsilon_{t}-\theta_{1} \varepsilon_{t-1}-\theta_{2} \varepsilon_{t-2}-\cdots-\theta_{4} \varepsilon_{t-4}
$$

For JKSE index:

$$
Y_{t}=\delta+\beta_{1} y_{t-1}+\beta_{2} y_{t-2}+\beta_{3} y_{t-3}+\varepsilon_{t}-\theta_{1} \varepsilon_{t-1}-\theta_{2} \varepsilon_{t-2}-\theta_{3} \varepsilon_{t-3}
$$

For Hang Seng index:

For Nikkei index:

$$
Y_{t}=\delta+\beta_{1} Y_{t-1}+\varepsilon_{t}-\theta_{1} \varepsilon_{t-1}-\theta_{2} \varepsilon_{t-2}
$$

$$
Y_{t}=\delta+\beta_{1} Y_{t-1}+\varepsilon_{t}
$$

Table 5

ARMA Forecasting Models Obtained for Stock Market Returns of Malaysia, Indonesia, Hong Kong, and Japan

\begin{tabular}{lllll}
\hline & Malaysia & Indonesia & Hong Kong & Japan \\
\cline { 2 - 5 } & ARMA $(3,4)$ & ARMA $(3,3)$ & ARMA $(1,2)$ & ARMA $(1,0)$ \\
\hline C & 0.005 & 0.116 & 0.0064 & -0.003 \\
AR(1) & -0.754 & -0.759 & -0.974 & 0.149 \\
AR(2) & 0.602 & -1.012 & - & - \\
AR(3) & 0.643 & -0.110 & - & - \\
MA(1) & 0.939 & 1.082 & 1.229 & - \\
MA(2) & -0.477 & 1.247 & 0.079 & - \\
MA(3) & -1.013 & 0.332 & - & - \\
MA(4) & -0.448 & - & - & - \\
\hline
\end{tabular}

To test the accuracy of the forecasted values by $\operatorname{ARMA}(p, q)$ model, dynamic, and static forecasts are then obtained as shown in Table 6 below.

Based on the values of RMSE as shown in Table 6, dynamic forecasts obtained for Malaysia, Indonesia and Hong Kong have lower values, while static forecast obtained for Japan has a lower value. This suggests that the calculated forecasts obtained for Malaysia, Indonesia, and Hong Kong for periods following the first period are obtained by employing a $n$-step ahead forecast procedure, while a 1-step ahead forecast procedure, which uses actual rather than the forecasted values, is employed for Japan. 
Table 6

Dynamic and Static Forecast

\begin{tabular}{llll}
\hline & RMSE & MAE & MAPE \\
\hline Malaysia & & & 80.7710 \\
\hline Dynamic & 0.0270 & 0.0210 & 91.8446 \\
Static & 0.0282 & 0.0226 & \\
\hline Indonesia & & 374.1801 \\
\hline Dynamic & 0.0495 & 0.0383 & 673.2236 \\
Static & 0.0633 & 0.0500 & \\
\hline Hong Kong & & 270.9018 \\
\hline Dynamic & 0.0551 & 0.0413 & \\
Static & 0.0587 & 0.0444 & 120.5353 \\
\hline Japan & & 146.0187 \\
\hline Dynamic & 0.0538 & 0.0425 & \\
Static & 0.0534 & 0.0425 &
\end{tabular}

\section{Exponentially Weighted Moving Average (EWMA) Model}

Based on JP Morgan's Risk Metrics models with the parameter of $97 \%$ for the monthly volatility estimations and declining in weightage from recent to oldest prices, the summation obtained from $\mathrm{R}^{2}(\mathrm{w})$ to form the Exponentially Weighted Moving Average (EWMA) value for individual markets are shown in Table 7 below.

Table 7

EWMA Weightage

\begin{tabular}{ll}
\hline & EMWA \\
\hline Malaysia & $1.3975 \%$ \\
Indonesia & $1.2615 \%$ \\
Hong Kong & $0.8626 \%$ \\
Japan & $0.3342 \%$ \\
\hline
\end{tabular}

\section{Generalized Auto-Regressive Conditional Heteroscedastic (GARCH)}

Using the general form of Generalized Auto-Regressive Conditional Heteroscedastic (GARCH) model below, results obtained for the $\operatorname{GARCH}(1,1)$ forecast are shown in Table 8.

Table 8

Evaluation of Forecasting Accuracy

\begin{tabular}{lllll}
\hline & Malaysia & Indonesia & Hong Kong & Japan \\
\hline $\mathrm{C}$ & 0.000126 & 0.000454 & 0.000167 & 0.00156 \\
$\mathrm{RESID}(-1)^{\wedge} 2$ & 0.057538 & 0.131799 & 0.156736 & 0.22425 \\
GARCH(-1) & 0.878522 & 0.805903 & 0.805512 & 0.3459 \\
\hline
\end{tabular}

Note. This table shows results obtained from the following $\operatorname{GARCH}(1,1)$ model: $\sigma_{\mathrm{t}}^{2}=\omega+\alpha \varepsilon_{\mathrm{t}-1}^{2}+\beta \sigma_{\mathrm{t}-1}^{2}$. Source: Results obtained by GARCH $(1,1)$ model.

As shown in Table 9, GARCH $(1,1)$ is found to be the best forecasting model for Malaysia, Indonesia, and Japan, while EWMA is found to be the best forecasting model for Hong Kong. The different forecasting model-EWMA - rather than GARCH $(1,1)$ found for Hong Kong could be due to the fact that Hong Kong is a net-contributor, whereas Japan a net-recipient. 
Table 9

Forecasting Accuracy Across Forecast Models

\begin{tabular}{llll}
\hline & EWMA & ARMA & GARCH \\
\hline Malaysia & & & 0.0007 \\
\hline MSE & 0.0014 & 0.0016 & 0.0208 \\
MAE & 0.0290 & 0.0311 & 0.0263 \\
RMSE & 0.0376 & 0.0405 & 1 \\
\hline Rank & 2 & 3 & \\
\hline Indonesia & & & 0.0021 \\
\hline MSE & 0.0039 & 0.0057 & 0.0363 \\
MAE & 0.0485 & 0.0626 & 0.0453 \\
RMSE & 0.0628 & 0.0758 & 1 \\
\hline Rank & 2 & 3 & 0.0029 \\
\hline Hong Kong & & & 0.0407 \\
\hline MSE & 0.0000 & 0.0052 & 0.0542 \\
MAE & 0.0012 & 0.0547 & 2 \\
RMSE & 0.0016 & 0.0719 & \\
\hline Rank & 1 & 3 & 0.0028 \\
Japan & & 0.0029 & 0.0527 \\
\hline MSE & 0.0051 & 0.0426 & 1 \\
MAE & 0.0596 & 0.0535 & \\
RMSE & 0.0717 & 2 & \\
Rank & 3 & &
\end{tabular}

\section{Conclusion}

In this study, using index return series obtained for Malaysia (FTSE KLCI), Indonesia (JKSE), Hong Kong (Hang Seng), and Japan (Nikkei), three forecasting models, ARIMA, EWMA, and GARCH $(1,1)$, were employed. The obtained results confirm that none of the chosen forecasting models appears be robust for all stock markets in the sample. In addition, the higher volatilities present in Hong Kong market as compared to that in Malaysia suggest that developed stock markets can have higher volatilities than those in emerging markets. Based on the lowest forecasting errors finding for MAE, MSE, RMSE, GARCH $(1,1)$ model was found to be a robust model for all three stock markets in Malaysia, Indonesia, and Japan and EWMA model which was found to be a better model for Hong Kong stock market. In the case of Malaysia, results obtained for this study are consistent with the findings in Angabini and Wasiuzzaman (2010), Kosapattarapim, Lin and Mccrae (2011) and Lim and Sek (2013) that the appropriate for forecasting model for Malaysia stock market is GARCH $(1,1)$, as compared to other forecasting models. In overall, GARCH $(1,1)$ appears to be the better forecasting model for most stock markets in the sample, which confirms the claim made by Minkah (2007). As the study is confined to a sample of two emerging countries (Indonesia and Malaysia) and two developed countries (Japan and Hong Kong), results obtained may be different for other stock markets in Europe, North America, etc. Further research should be carried out to reconfirm the robustness of the GARH $(1,1)$ model in more stock markets from other continents rather than in Asia.

\section{References}

Adebayo, F. A., \& Sivasamy, R. (2014). Forecasting stock market series with ARIMA model, 3(3), 65-77. 
Angabini, A., \& Wasiuzzaman, S. (2010). Modeling the effects of the global financial crisis on the Malaysian market. International Journal of Trade, Economics and Finance, 1(4), 387-391.

Febrian, E. (2006). Modeling and forecasting Jakarta stock exchange: Stock market volatility. Working Paper.

Kosapattarapim, C., Lin, Y., \& McCrae, M. (2012). Evaluating the volatility forecasting performance of best fitting GARCH models in emerging Asian stock markets. International Journal of Mathematics \& Statistics, 12(2), 1-15.

Ladokhin, S. (2009). Forecasting volatility in the stock market, 12(1), 33.

Lim, C. M., \& Sek, S. K. (2013). Comparing the performances of GARCH-type models in capturing the stock market volatility in Malaysia. Procedia Economics and Finance, 5(13), 478-487.

Makridakis, S., Wheelwright, S. C., \& Hyndman, R. J. (1998). Forecasting methods and applications (3rd ed.), 18, 158-159. New York: Wiley.

Minkah, R. (2007). Forecasting volatility, 7(727), 1-61.

Schwert, G. (1990). Stock market volatility. Financial Analysts Journal, 46, 23-34.

Singh, G. (2015). Volatility modelling and forecasting for NIFTY stock returns. NMIMS Management Review, 16(3), 89-108.

Wilhelmsson, A. (2006). Garch forecasting performance under different distribution assumptions. Journal of Forecasting, 25(8), 561-578.

Tse, Y. K. (1991). Stock returns volatility in the Tokyo Stock Exchange. Japan and the World Economy, 3, 285-298. 